\title{
Impact of Social Defeat Stress on DNA Methylation in Drd2, Nr3c1, and Stmn 1 in Wild-type and Stmn1 Knock-out Mice
}

\author{
Young-Eun $\mathrm{Oh}^{1,2}$, Thong Ba Nguyen ${ }^{1,2}$, Fatima Zahra Rami ${ }^{1,2}$, Maryam Karamikheirabad ${ }^{1,2}$, Young-Chul Chung ${ }^{1,2}$ \\ ${ }^{1}$ Department of Psychiatry, Jeonbuk National University Medical School, ${ }^{2}$ Research Institute of Clinical Medicine of Jeonbuk National University, \\ Biomedical Research Institute of Jeonbuk National University Hospital, Jeonju, Korea
}

Objective: Epigenetic profiles can be modified by stress. Dopamine receptor D2 (Drd2), glucocorticoid receptor gene ( $\mathrm{Nr} 3 \mathrm{c} 1)$ and Stathmin 1 (Stmn1) genes are all implicated in adaptation to stress. The aim of study is to investigate impact of social defeat on DNA methylation in Drd2, Nr3c1, and Stmn1 in wild-type (WT) and Stmn1 knock-out (KO) mice.

Methods: The WT and Stmn1 KO mice were subjected to chronic social defeat. Brain tissues of the prefrontal cortex (PFC), amygdala (AMY) and hippocampus (HIP) were obtained. We measured DNA methylation levels of the Drd2, $\mathrm{Nr3c1}$, and Stmn1 genes in the PFC, AMY, and HIP using pyrosequencing.

Results: In WT mice, social defeat stress did not induce any changes in Drd2 methylation, whereas significant hypermethylation occurred in $\mathrm{Nr} 3 \mathrm{c} 1$ and Stmn1 in the susceptible and unsusceptible groups, respectively, compared to the control group. The methylation responses in the Stmn1 KO mice differed from those seen in the WT mice, such that hypermethylation was evident in all three genes in the susceptible and unsusceptible groups compared to control group. Comparison of the Stmn1 KO and WT mice revealed the same pattern of hypermethylation for all three genes. Conclusion: Social defeat stress induced different epigenetic modifications in three genes among control, unsusceptible, and susceptible groups of WT and Stmn1 KO mice. In particular, hypermethylation of Nr3c1 in the HIP of the susceptible group, and of Stmn1 in the AMY of the unsusceptible group in WT mice, could serve as epigenetic biomarkers of stress susceptibility and stress resilience, respectively.

KEY WORDS: Social defeat stress; DNA methylation; Dopamine receptor D2; Nr3c1; Stathmin 1; Epigenetic.

\section{INTRODUCTION}

Epigenetics can be defined as alterations in phenotype or gene expression due to mechanisms other than changes in the underlying DNA sequence. This phenomenon is known to reflect the sensitivity and responsiveness of animal and human brains to constantly changing circumstances that regulate gene expression profiles, and involves covalent modification of DNA (DNA methylation) as well as the acetylation, methylation, and phosphorylation of histones and DNA-associated proteins. DNA methylation occurs through 5-methyl- and 5-hydrox-

Received: September 17, 2020 / Revised: October 26, 2020

Accepted: October 27, 2020

Address for correspondence: Young-Chul Chung

Department of Psychiatry, Jeonbuk National University Medical

School, 20 Geonji-ro, Deokjin-gu, Jeonju 54907, Korea

E-mail: chungyc@jbnu.ac.kr

ORCID: https://orcid.org/0000-0001-9491-1822 ymethylcytosine ( $5 \mathrm{mC}$ and $5 \mathrm{hmC}$, respectively) and is considered to be among the principal interfaces between the genome and the environment; thus, epigenetics can help explain phenotypic variations.

Social defeat is defined as an individual losing a confrontation among conspecific animals in either a dyadic or group context. Adaptation to social defeat-induced stress is an important component of social homeostasis; maladaptation during this process may have pathological sequelae. The social defeat stress model is considered important for investigating the effects of environmental factors on behavior, and can provide important insights into the determinants of vulnerability or resilience to stress. Early life adversity (ELA) is the most frequently used paradigm for investigating the effects of stress on DNA methylation. Key findings from ELA studies include increased levels of DNA methylation in BDNF in the adult

(c) This is an Open-Access article distributed under the terms of the Creative Commons Attribution Non-Commercial License (http://creativecommons.org/licenses/by-nc/4.0) which permits unrestricted non-commercial use, distribution, and reproduction in any medium, provided the original work is properly cited. 
rat prefrontal cortex (PFC) [1], hypomethylation of the $\mathrm{Nr} 3 \mathrm{c} 1$ promoter in the rat hippocampus (HIP) [2], and hypomethylation of the arginine vasopressin enhancer region in the mouse hypothalamic paraventricular nucleus (PVN) [3]. However, to date, only a single study [4] has investigated the effects of social defeat stress on DNA methylation in mice. These authors reported that defeat stress induces demethylation of the corticotrophin-releasing factor promoter region.

In the present study, three genes of interest associated with DNA methylation were investigated: dopamine receptor D2 (Drd2), nuclear receptor subfamily 3, group C, member 1 (Nr3c1), and stathmin 1 (Stmn1). The dopamine D2 receptor is closely associated with locomotion, reward, and memory [5]. Stress directly influences several fundamental behaviors and phenomena that are mediated by the dopaminergic system, including locomotor activity, sexual activity, appetite, and cross-sensitization with drugs of abuse [6]. The regulation of $\mathrm{Nr} 3 \mathrm{c} 1$, which is a glucocorticoid receptor (GR) gene, is important for adaptation to stress [7]. Stmn 1 produces a protein that is critical for microtubule (MT) polymerization, and is also involved in fear processing in both mice [8] and humans [9]. Given that Stmn1 knock-out (KO) mice display anxious hyperactivity, impaired recognition and decreased levels of neutral behavior compared to wild-type (WT) mice [10], we hypothesized that impact of social defeat would be greater in Stmn1 KO mice.

The aim of study was to determine the consequences of chronic social defeat stress on the DNA methylation profiles of the putative promoter and first intron regions of the Drd2 and Stmn1 genes, and of the exon $1_{7} \mathrm{Nr} 3 \mathrm{c} 1$ promoter, in WT and Stmn1 KO mice. In particular, DNA methylation levels were compared among control, un- susceptible (UNS), and susceptible (SUS) groups of WT and Stmn1 KO mice, and between the genotypes (WT vs. Stmn1 KO mice) within each group.

\section{METHODS}

\section{Experimental Animals}

To breed the Stmn 1 KO mice on a C57BL/6J background, three heterozygous $\mathrm{C} 57 \mathrm{BL} / 6 \mathrm{~J}$ females and males were purchased from Jackson Laboratory (strain name:

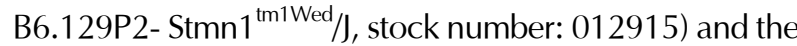
line was maintained by successively backcrossing heterozygotes $\left(\mathrm{Stmn}^{+/-}\right.$; two female mice and one male mouse). Genotyping was performed as described previously [11]. All experiments were conducted using male homozygous Stmn 1 KO and WT mice, born from heterozygous mutants that were maintained on the C57BL/6 background. All procedures were conducted in strict accordance with the guidelines for animal experiments of the Institutional Animal Care and Use Committee (IACUC) of Jeonbuk National University and the National Institutes of Health $(\mathrm{NIH})$ principles for the Care and Use of Laboratory Animals based on the 3Rs (replacement, refinement, and reduction; NIH Pub. No. 85-23, revised in 1996). The entire project was reviewed and approved by the IACUC (cuh-IACUC-151027-32) of Jeonbuk National University Medical School). A schematic of the experimental design is shown in Figure 1.

\section{Procedure for Inducing Social Defeat Stress}

The mice were exposed to social defeat stress via the resident-intruder paradigm. Specifically, both genotypes ( $n=20$ for each) of mice underwent 10 days of social defeat stress via confrontations with an aggressive, larger CD

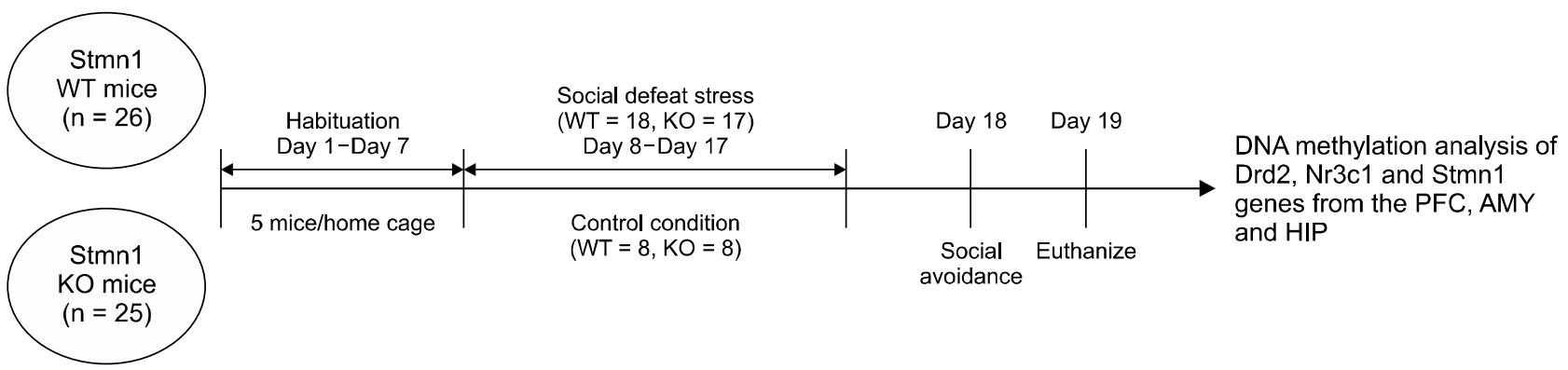

Fig. 1. Experimental design and its timeline.

Drd2, dopamine receptor D2; KO, knock-out; $\mathrm{Nr3c1}$, nuclear receptor subfamily 3 group C member 1; Stmn1, stathmin 1; WT, wild type. 
1 mouse that was approximately 16 weeks old. All male CD1 mice were screened for aggressiveness by measuring the latency to attack a naive C57BL/6J mouse. Only CD1 mice that attacked in less than 60 seconds in at least two consecutive sessions during three 180-second screening sessions were used; this equated to $15 \%$ of the screened aggressors.

The $\mathrm{C} 57 \mathrm{BL} / 6 \mathrm{~J}$ mice were introduced into the home cage of the unfamiliar CD1 aggressor mouse and allowed to interact for 5 minutes. During this period, all subject mice were defeated and showed signs of subordination (i.e., lying on their back and/or freezing or adopting upright submissive postures). After 10 minutes of full interaction, the defeated mouse was separated from the aggressive resident by inserting a perforated Plexiglas divider into the cage (which also allowed for sensory contact for the rest of the day). On the subsequent day, the C57BL/6J mouse was exposed to a new resident CD1 aggressor mouse to prevent habituation. The social defeat procedure lasted for 10 consecutive days. As a control group, C57BL/6J mice ( $\mathrm{n}=8$ for each genotype) were placed into equivalent cages with members of the same strain, which were changed daily.

\section{Social Avoidance Test}

Following completion of the social defeat procedure, the social avoidance test was performed on day 11 of the study, to categorize the mice into UNS and SUS groups. Each defeated mouse was placed into an interaction box $(42 \times 42 \mathrm{~cm})$ that consisted of a wire mesh cage $(10 \times 4.5$ $\mathrm{cm})$ located at one end and an interaction zone $(8-\mathrm{cm}$ wide) surrounding the cage. The test comprised two sessions, separated by a 1-minute interval. In the first session, no CD1 mouse was present in the wire mesh cage and the movement of the defeated animal was tracked for 2.5 minutes. In the second session, a novel CD1 mouse was introduced into the wire mesh cage and the same defeated animal from the first session was placed into the box and tracked for another 2.5 minutes. The total time spent by the experimental mouse in the 8 -cm-wide corridor surrounding the wire mesh cage (interaction zone) was calculated automatically using SMART software (Panlab, Barcelona, Spain) and a social interaction (SI) ratio was derived as follows: $100 \times$ (interaction time with target mouse present) / (interaction time with no target mouse present). Based on previous studies [12,13], a SI ratio of 100 was used as the cut-off value, such that scores $<100$ were defined as "susceptible" and scores $\geq 100$ were defined as "unsusceptible".

\section{Brain Tissue Collection}

After segregation into the UNS and SUS groups, the mice were euthanized via cervical dislocation. Subsequently, the PFC and HIP were dissected using microspatulas and the amygdala (AMY) was punched out on an ice plate using a 1-mm Harris Uni-Core micro-punch (Electron Microscopy Science, Hatfield, PA, USA). The tissues (15-18 $\mathrm{mg}$ of the PFC, 4-5 mg of the AMY, and 18-22 mg of the HIP) were quickly cryopreserved in liquid nitrogen and stored at $-80^{\circ} \mathrm{C}$ until assay.

\section{DNA Methylation Analysis}

\section{DNA extraction and bisulfite treatment}

DNA samples from the PFC, AMY, and HIP were extracted using DNase Blood \& Tissue Kits (QIAGEN, Hilden, Germany) according to the manufacturer's protocol. Subsequently, bisulfite conversion of $500 \mathrm{ng}$ of genomic DNA was achieved using the EpiTect bisulfite kit (QIAGEN) according to the manufacturer's instructions.

\section{Bisulfite pyrosequencing}

DNA methylation was measured by pyrosequencing the polymerase chain reaction (PCR) products. Primers were designed against the putative promoter and first intron regions of the Drd 2 and Stmn 1 genes, which were assumed to be located from $-1 \mathrm{~kb}$ to $+500 \mathrm{bp}$ of the transcription start site (TSS). For $\mathrm{Nr} 3 \mathrm{c} 1$, a primer was designed against the exon 1 region of the GR (chr18:39,489,956$39,490,734)$ [14], which has been extensively studied with regard to stress. Several regions that were 779-901 bp long (801, 779, and 901 bp for Drd2, Nr3c1, and Stmn1, respectively) were initially designed using PyroMark Assay Design 2.0 software (QIAGEN). Afterwards, key regions, i.e., those that had more transcription factor binding sites were selected using JASPAR (http://jaspar.genereg.net/). Details about the PCR primers and sequencing primer are shown in Supplementary Table 1.

Next, $40 \mathrm{ng}$ of bisulfite-treated DNA was amplified in a $25-\mu$ l reaction volume using the GeneAmp PCR System 9700 (Applied Biosystems, Foster City, CA, USA). Either the forward or reverse primer was biotinylated to convert 
the PCR product to single-stranded DNA templates, and a sequencing primer that annealed to the single-stranded DNA template was then added [15]. The amplification of the PCR step consisted of 40 cycles $\left(94^{\circ} \mathrm{C}\right.$ for 1 minute, 58 $60.5^{\circ} \mathrm{C}$ for 30 seconds, and $72^{\circ} \mathrm{C}$ for 1 minute); the primer sets, locations, and PCR conditions for each region are presented in Supplementary Table 1. The pyrosequencing reactions were performed in a PyroMark Q48 Autoprep system (QIAGEN) and quantification of the CpG methylation (percentage of the relative light unit [RLU] of the $\mathrm{C}$ peak [methylated cytosine]/RLU of C peak $+\mathrm{T}$ peak [unmethylated cytosine]) was performed with PyroMark Q48 Autoprep 2.4.2 software (QIAGEN). When the peak value of a base exceeded $20 \mathrm{RLU}$, the pyrosequencing results were considered to be reliable.

Histogram results were reanalyzed if they did not meet the following criteria: 1) in the overlapped histogram of the expected and actual results, the a) magnitude of the RLU difference of any mismatched peaks among all samples was > $20 \mathrm{RLU}, \mathrm{b}$ ) the background peak was inconsistent among the samples and the RLU of a background peak was $>7 \%$ of the mean RLU of a single peak, and c) the analysis for a certain $\mathrm{CpG}$ site failed due to the preceding polybases ( $\geq$ three identical bases) and the peak heights among all samples for that CpG site were inconsistent; and 2) any peak showed a double-peaked structure.

\section{Statistical Analysis}

Shapiro - Wilk tests of the normality of the data were all non-significant. Two-way analysis of variance (ANOVA) was conducted to assess the interaction and main effects of genotype and group (Supplementary Tables 2, 5-10) ; $t$ tests or additional ANOVAs were performed to further explore differences between genotypes or among groups. The methylation data of both the individual and combined CpGs were included in the analyses, but only the results for the latter are described and discussed (see Supplementary Tables 11-22 for the individual CpG results). Post hoc analyses, including Tukey's honestly significant difference tests and Pearson's correlation analyses, were performed to assess the relationships between the SI ratio and DNA methylation levels of each gene. All results are presented as mean \pm standard error of the mean and all data were analyzed using R software (ver. 3.5.3; R Development Core Team, Vienna, Austria). In all cases, $p$ values $\leq 0.05$ was considered to indicate statistical significance.

\section{RESULTS}

\section{Main and Interaction Effects for the Three Genes}

For Drd2, two-way ANOVA revealed a significant effect of genotype $\left(\mathrm{F}_{[1,42]}=4.690, p=0.036\right)$ on the DNA methylation levels of the combined CpGs in the HIP, and a significant effect of group $\left(\mathrm{F}_{[2,42]}=3.503, p=0.039\right)$ on the DNA methylation levels of the combined CpGs in the AMY. For $\mathrm{Nr} 3 \mathrm{c} 1$, there were significant effects of group on the DNA methylation levels of the combined CpGs in the $\operatorname{PFC}\left(\mathrm{F}_{[2,42]}=4.590, p=0.016\right)$ and $\operatorname{HIP}\left(\mathrm{F}_{[2,42]}=5.272\right.$, $p=0.009$ ) as well as a significant group $\times$ genotype interaction effect $\left(\mathrm{F}_{[2,42]}=4.084, p=0.024\right)$ on the DNA methylation levels of the combined CpGs in the HIP. For Stmn1, there were significant effects of group on the DNA methylation levels of the combined CpGs in the PFC $\left(\mathrm{F}_{[2,42]}=4.721, p=0.014\right)$. Within the AMY and HIP, there were significant main effects of genotype on the DNA methylation levels of the AMY $\left(\mathrm{F}_{[1,42]}=14.034, p=\right.$ $0.001)$ and $\operatorname{HIP}\left(\mathrm{F}_{[1,42]}=20.562, p<0.001\right)$, significant main effects of group on the DNA methylation levels of the $\operatorname{AMY}\left(\mathrm{F}_{[2,42]}=6.031, p=0.005\right)$ and $\operatorname{HIP}\left(\mathrm{F}_{[2,42]}=\right.$ $5.598, p=0.007)$, and a significant group $\times$ genotype in teraction effect on the DNA methylation levels of the AMY $\left(\mathrm{F}_{[2,42]}=6.197, p=0.004\right)$ and $\operatorname{HIP}\left(\mathrm{F}_{[2,42]}=7.677, p=\right.$ 0.001; Supplementary Table 2).

\section{Methylation Levels in the Three Groups of WT and Stmn 1 KO Mice}

There were significant differences in the DNA methylation levels of the combined $\mathrm{CpGs}$ in $\mathrm{Nr} 3 \mathrm{c} 1$ in the HIP $\left(\mathrm{F}_{[2,21]}=5.818, p=0.010\right)$ among the three WT mouse groups. Post hoc tests revealed significantly higher DNA methylation levels of the combined CpGs in the SUS group compared to the control $(p=0.043)$ and UNS groups $(p=0.011)$. There was also a significant difference in the DNA methylation levels of the combined CpGs in Stmn 1 in the AMY $\left(\mathrm{F}_{[2,21]}=16.430, p<0.001\right)$ among the three WT groups. Post hoc tests revealed significantly higher DNA methylation levels of the combined CpGs in the UNS group compared to the control $(p<0.001)$ and SUS groups ( $p<0.001$; Fig. 2 and Supplementary Table 3 ). There was a significant difference in the DNA methyl- 

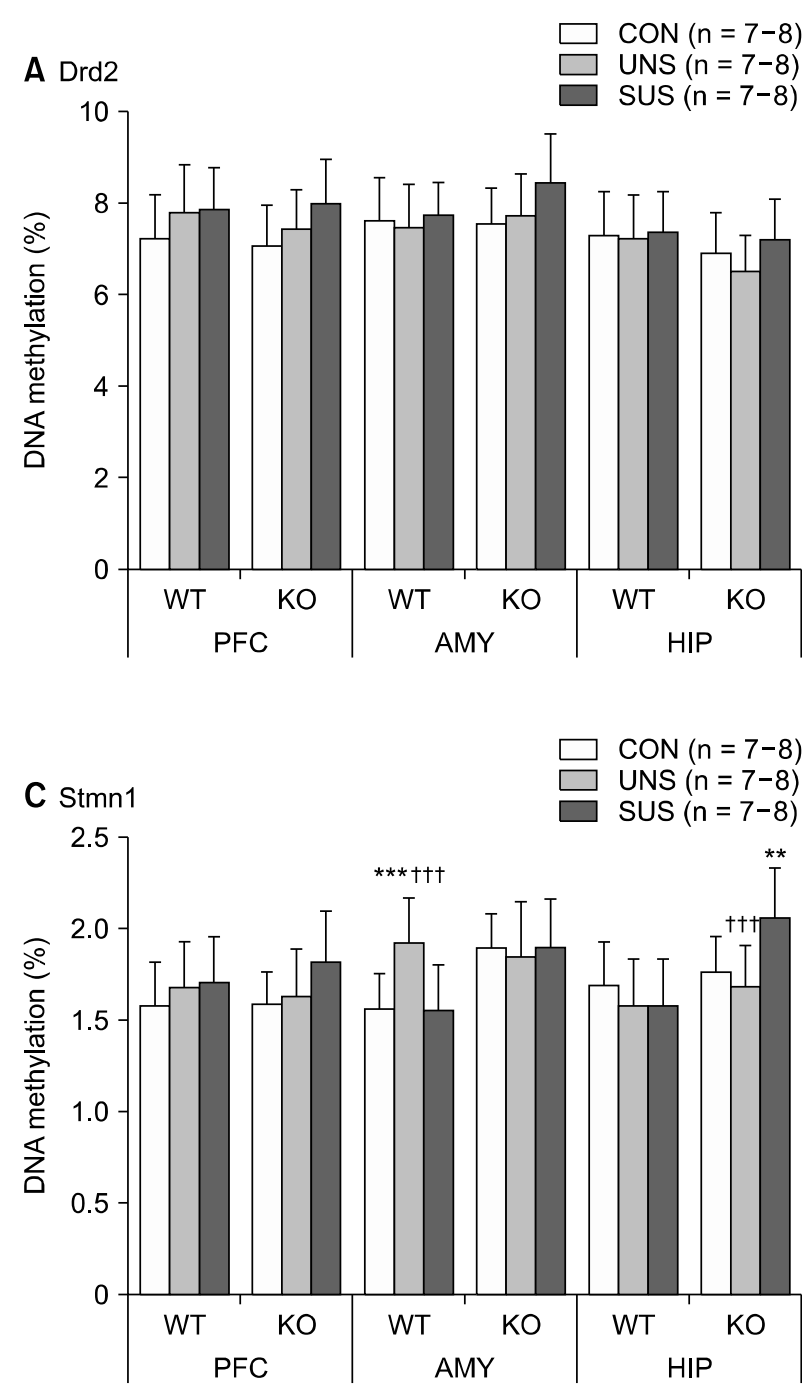

ation levels of combined CpGs in Drd2 in the AMY $\left(\mathrm{F}_{[2,21]}=\right.$ 3.634, $p=0.044$ ) among the three Stmn $1 \mathrm{KO}$ mouse groups. Post hoc tests revealed significantly higher DNA methylation levels in the SUS group $(p=0.050)$ compared to the control group. There was also a significant difference in the DNA methylation levels of the combined CpGs in $\mathrm{Nr} 3 \mathrm{c} 1$ in the $\operatorname{HIP}\left(\mathrm{F}_{[2,21]}=4.128, p=0.031\right)$ among the three $\mathrm{KO}$ groups. Post hoc tests revealed significantly higher DNA methylation levels of the combined CpGs in the UNS group $(p=0.032)$ compared to the control group. For Stmn1, there were significant differences in the DNA methylation levels of the combined CpGs in the $\operatorname{PFC}\left(\mathrm{F}_{[2,21]}=3.533, p=0.048\right)$ and $\operatorname{HIP}\left(\mathrm{F}_{[2,21]}=10.880, p=\right.$ $0.001)$ among the three KO groups. Post hoc tests revealed significantly higher DNA methylation levels of the combined CpGs in the SUS group compared to the con-

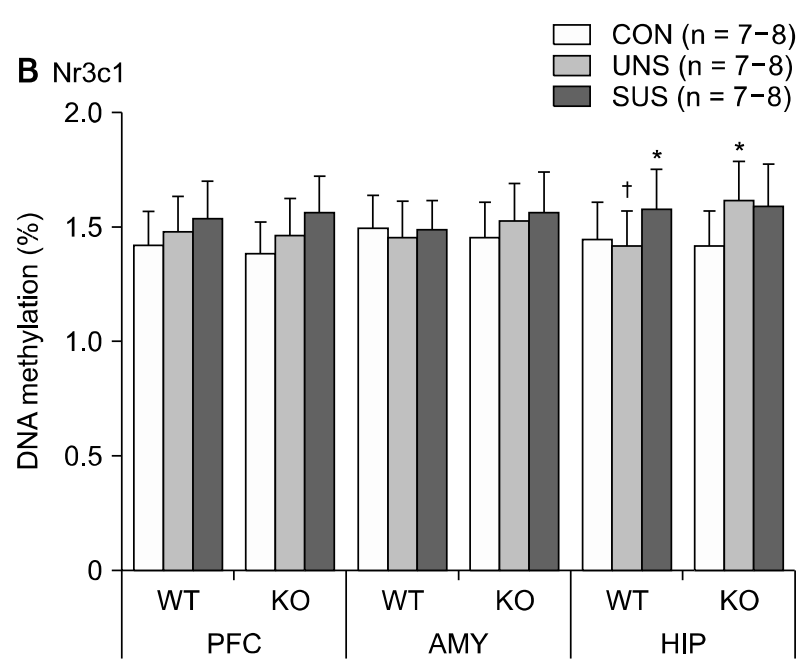

Fig. 2. Methylation percentage of the combined $\mathrm{CpG}$ in the (A) Drd2, (B) Nr3c1, and (C) Stmn1 genes among the three groups of the WT and Stmn1 KO mice.

Drd2, dopamine receptor D2; Stmn1, stathmin 1; KO, knock-out; WT, wild type; CON, control; UNS, unsusceptible; SUS, susceptible; PFC, prefrontal cortex; AMY, amygdala; HIP, hippocampus.

${ }^{*} p<0.05,{ }^{* *} p<0.01,{ }^{* * *} p<0.001$ vs. control group by Tukey's HSD test. ${ }^{\dagger} p<0.05,{ }^{+{ }^{+}+} p<0.001$ vs. susceptible group by Tukey's HSD test.

trol $(p=0.006)$ and UNS $(p=0.001)$ groups in the HIP. However, post hoc tests for the PFC did not reveal any significant group differences (Fig. 2 and Supplementary Table 3).

\section{Comparison of Methylation Levels between the WT and Stmn 1 KO Mice in Each Group}

In the control group, there was a significant increase in the methylation level of the combined CpGs in Stmn1 in the AMY of Stmn1 KO mice ( $p=0.002)$ compared to the WT mice. In the UNS group, there was a significant increase in the methylation level of the combined CpGs in $\mathrm{Nr} 3 \mathrm{c} 1$ in the HIP of the Stmn1 KO mice ( $p=0.012)$ compared to the WT mice. In the SUS group, there was a significant increase in the methylation level of the combined CpGs in Drd2 in the AMY of the Stmn1 KO mice $(p=$ 

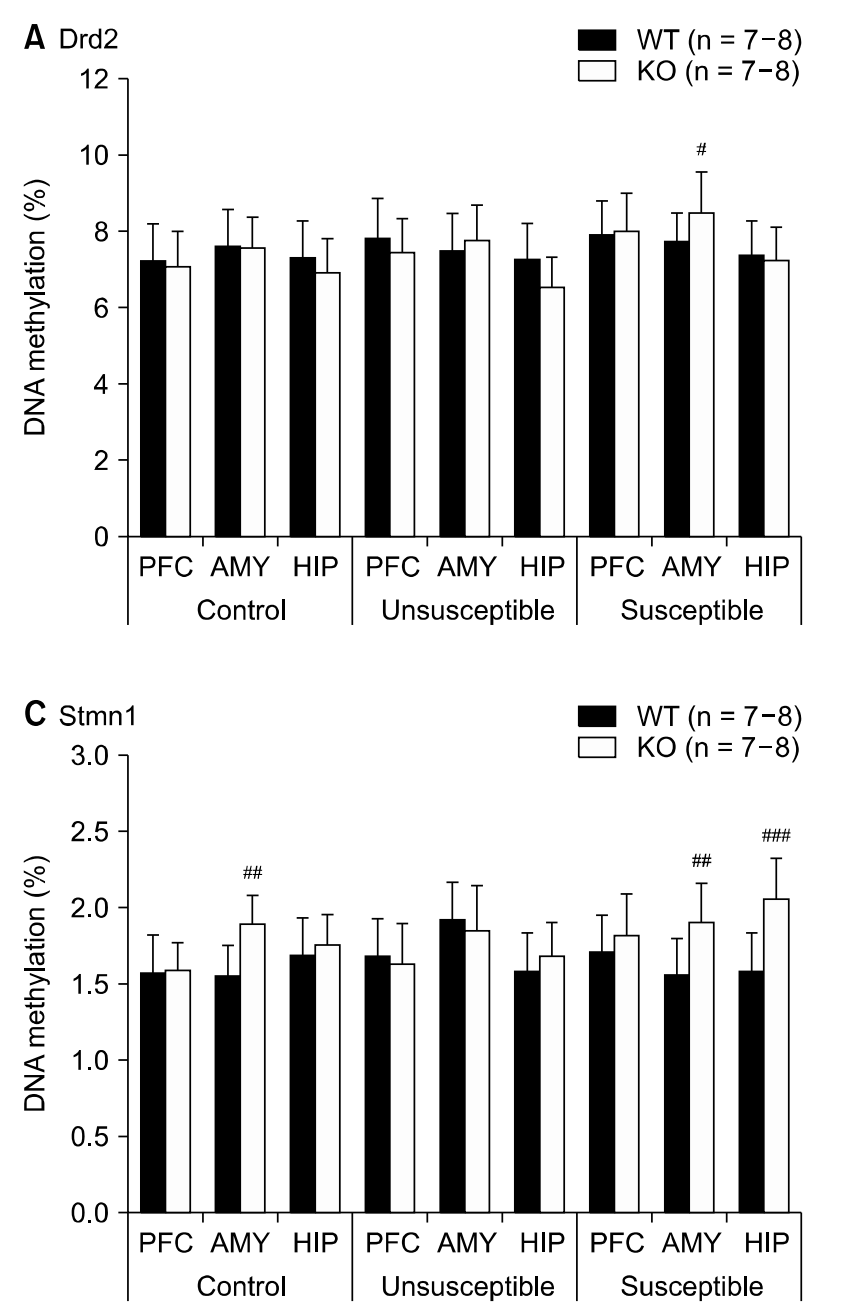

0.021) compared to the WT mice. Additionally, there was a significant increase in the methylation level of the combined CpGs in Stmn1 in the AMY of Stmn1 KO mice ( $p=$ 0.006) compared to the WT mice. In the HIP, there was a significant increase in the methylation level of the combined CpGs in Stmn1 $(p<0.001)$ of the Stmn1 KO mice compared to the WT mice (Fig. 3 and Supplementary Table 4).

\section{Correlation Analysis}

There were significant negative correlations between the SI ratio and DNA methylation levels of region 4 in $\operatorname{Drd} 2(r=-0.56, p=0.005)$ and Nr3c1 $(r=-0.46, p=$ $0.025)$ in the PFC of WT mice. The analyses also revealed that the SI ratio and DNA methylation levels had a negative correlation in region 4 in Drd2 $(r=-0.43, p=$ $0.034)$, and a positive correlation in Stmn $1(r=0.58, p=$ 0.003), in the AMY of WT mice (Fig. 4). In Stmn1 KO

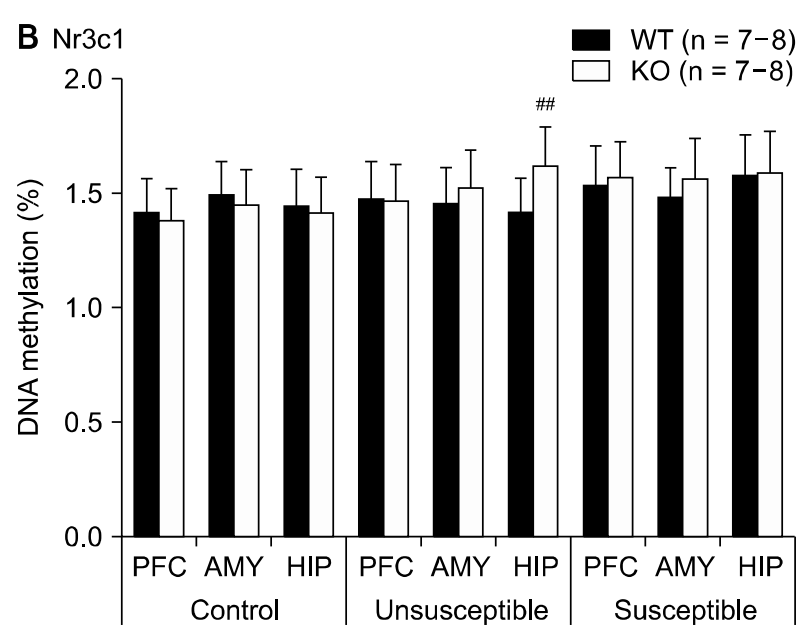

Fig. 3. Methylation percentage of the combined $\mathrm{CpG}$ in the (A) Drd2, (B) Nr3c1, and (C) Stmn1 genes between the WT and Stmn1 KO mice in each group.

Drd2, dopamine receptor D2; Stmn1, stathmin 1; KO, knock-out; WT, wild type; PFC, prefrontal cortex; AMY, amygdala; HIP, hippocampus.

${ }^{\#} p<0.05,{ }^{\# \#} p<0.01,{ }^{\# \# \#} p<0.001$ compared with the WT mice by $t$ test.

mice, there were negative correlations between the SI ratio and DNA methylation levels of region $4(r=-0.45, p=$ 0.026), and region 5 in $\operatorname{Drd} 2(r=-0.46, p=0.022)$, and of the combined CpGs $(r=-0.55, p=0.005)$ in Stmn 1 in the HIP (Fig. 5).

\section{DISCUSSION}

Epigenetic mechanisms have been implicated in a variety of processes through which social stressors harm the health of animals and humans. For example, DNA methylation that occurs through $5 \mathrm{mC}$ and $5 \mathrm{hmC}$ is considered to be one of the principal interfaces between the genome and environment, can therefore help to explain phenotypic variations. The present study was conducted to investigate the effects of social defeat stress on the DNA methylation patterns of three genes of interest in WT and Stmn1 KO mice. 


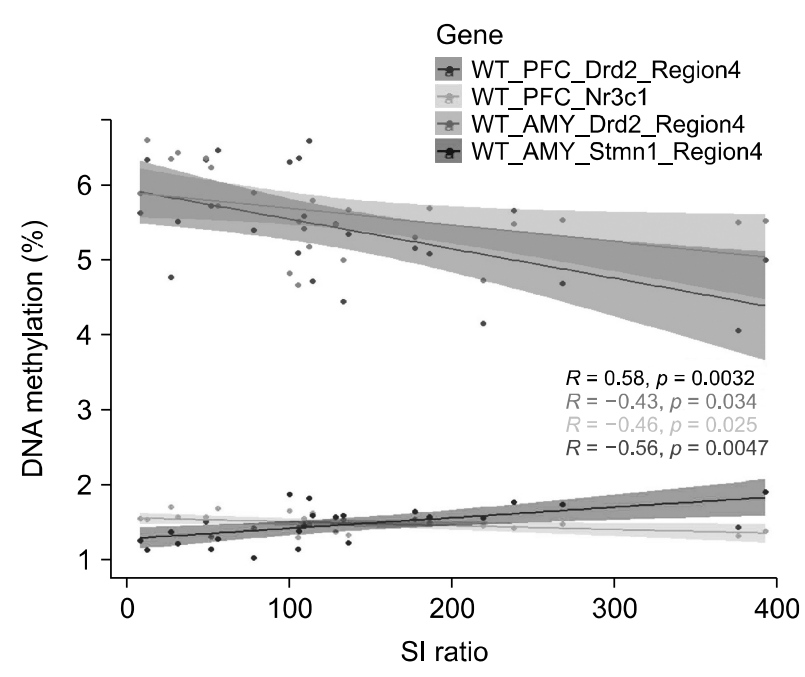

Fig. 4. Correlation plots depicting relationship between social interaction $(\mathrm{SI})$ ratio and DNA methylation of the Drd2 and $\mathrm{Nr} 3 \mathrm{c} 1$ genes in the PFC, and Drd 2 and Stmn 1 genes in the AMY of the WT mice. Drd2, dopamine receptor D2; Stmn1, stathmin 1; WT, wild type; PFC, prefrontal cortex; AMY, amygdala.

Comparison of the methylation percentages in Drd2 among the three WT groups revealed no significant difference in any brain region. Our research group previously observed no changes in Drd2 protein expression in defeated mice, in the same brain regions as those studied herein, following social defeat stress [13]. Although controversial, it has been hypothesized that methylation in region 4 of Drd2, which includes the first intron, is associated with downregulation of gene expression $[16,17]$. Hence, the present results showing no difference in methylation levels in Drd2 among the three WT groups appear consistent with previous findings regarding Drd2 expression [13]. Moreover, this finding (i.e., the lack of any difference among the control, UNS, and SUS groups) may indicate that DNA methylation in Drd2 does not contribute to phenotypic variations. Regarding $\mathrm{Nr} 3 \mathrm{c} 1$ in WT mice, there were significantly higher methylation levels in the HIP of the SUS group compared to the control group. This finding is not surprising, given that previous studies employing various stress paradigms, such as low maternal care or maternal separation, observed increased methylation levels in the exon ${ }_{17} \mathrm{Nr} 3 \mathrm{C} 1$ promoter in the HIP [18] and PVN [19]. Taken together, these findings suggest that methylation levels in the exon $1_{7} \mathrm{Nr} 3 \mathrm{c} 1$ promoter could serve as an epigenetic biomarker of stress susceptibility. It is also important to note that there were no differences in methylation levels between the UNS and control groups

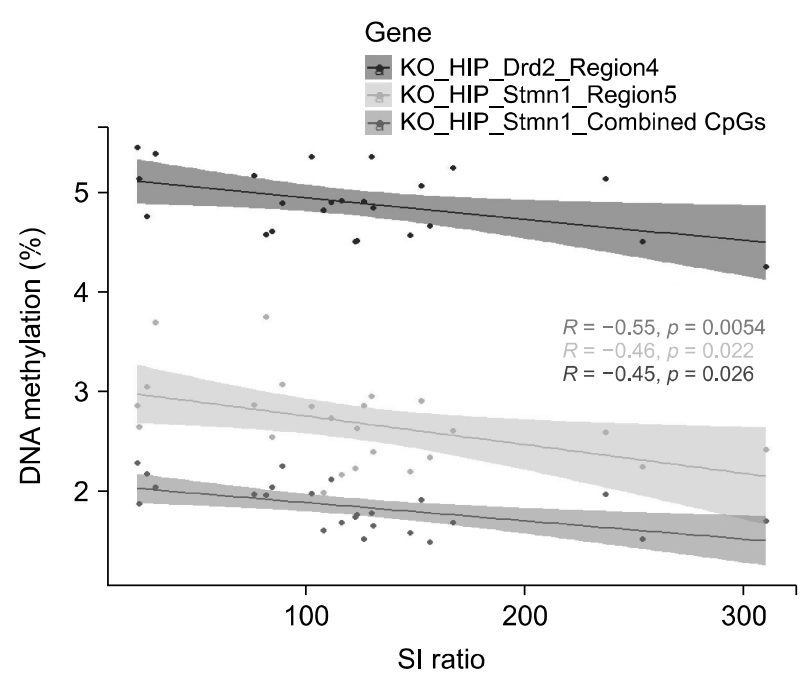

Fig. 5. Correlation plots depicting relationship between social interaction (SI) ratio and DNA methylation of the Drd2 and Stmn1 genes in the HIP of the Stmn1 KO mice.

Drd2, dopamine receptor D2; Stmn1, stathmin 1; KO, knock-out; HIP, hippocampus.

in the present study, which suggests that stress resilience was associated with certain factors that prevented enhanced methylation of the exon $1_{7} \mathrm{Nr} 3 \mathrm{c} 1$ promoter or other related processes. Interestingly, hypermethylation of Stmn1 was observed only in the AMY of the UNS group relative to the control and SUS WT groups. It has been shown that lower Stmn1 activity levels increase MT stability, which is involved in axonal growth, synaptic plasticity, neuronal differentiation, and memory [20], and modulates deficiencies in long-term potentiation in the AMY in response to defeat stress-induced fear [8]. Assuming that hypermethylation of Stmn1 results in decreased Stmn1 protein expression, the increased methylation of Stmn 1 in response to social defeat stress could be an appropriate epigenetic biomarker of stress resilience.

In Stmn1 KO mice, comparison of the Drd2 methylation percentages among the three groups, in all three brain regions, revealed significantly higher methylation levels only in the AMY of the SUS group compared to the control group. Our research group previously reported no changes in the expression levels of short- or long-form Drd2, among the same three groups and brain regions under investigation in this study, in Stmn1 KO mice [10]; this is inconsistent with the methylation data of the present study. It is possible that processes other than methylation influenced the present results; furthermore, the methylation patterns in the Stmn1 KO mice in response to defeat 
stress appeared to differ from those observed in WT mice. Regarding $\mathrm{Nr} 3 \mathrm{c} 1$ in Stmn1 $\mathrm{KO}$ mice, there were significantly higher levels of methylation in the HIP of the UNS group compared to the control group. From the perspective of stress resiliency, this finding contradicts previous results, because decreased GR expression due to enhanced methylation of $\mathrm{Nr} 3 \mathrm{c} 1$ may reduce glucocorticoid-mediated negative feedback to the hypothalamus and pituitary, which would in turn lead to persistent activation of the HPA axis and the manifestation of certain disorders $[21,22]$. It is possible that our UNS Stmn1 KO mouse group was more resilient due to factors other than $\mathrm{Nr} 3 \mathrm{c} 1$ methylation, even though they were more susceptible to defeat stress, at least in terms of $\mathrm{Nr} 3 \mathrm{c} 1$ methylation. Regarding Stmn1, the SUS group showed a trend toward higher methylation levels in the PFC compared to the control group, whereas in the HIP, significantly higher methylation levels were observed in the SUS group compared to the UNS and control groups. Assuming that enhanced methylation of Stmn1 reduces its expression levels, and that this change is beneficial for axonal growth, synaptic plasticity, and the fear extinction response [23], these findings are difficult to interpret. It is possible that the methylation of Stmn 1 seen in the SUS group of Stmn 1 KO mice differed from that in the SUS group of WT mice, because this process represents a compensatory mechanism against a pathophysiology that has already occurred in Stmn1 KO SUS mice [24]. Alternatively, it may merely represent an epiphenomenon.

Comparison of the methylation patterns of the three genes between the WT and $\mathrm{KO}$ control group mice did not reveal any significant differences in $\mathrm{Drd} 2$ or $\mathrm{Nr} 3 \mathrm{c} 1$, which suggests that there was no basal difference in the methylation levels of $\mathrm{Drd} 2$ and $\mathrm{Nr} 3 \mathrm{c} 1$ between the two genotypes. However, there was a significantly higher level of methylation in Stmn 1 in the AMY of the Stmn $1 \mathrm{KO}$ mice compared to the WT mice. The Stmn 1 KO mice were generated by replacing a genomic fragment that contained exons 2 and 3 with a neomycin selection cassette, which allowed for observation of changes in the methylation level of Stmn1. This finding suggests that, even in the absence of Stmn 1 protein expression, there appears to be a mechanism driving further decreases in Stmn1 protein expression in Stmn1 KO mice; however, the nature of this mechanism remains to be elucidated. In the UNS and SUS groups, all three genes exhibited higher methylation levels in Stmn1 KO mice than in WT mice, which suggests that the DNA methylation responses in the KO mice differed from those in the WT mice for all three genes, regardless of whether they were classified as UNS or SUS. However, how these differences relate to the behavioral characteristics of Stmn 1 KO mice remains largely unknown and merit further study.

Correlation analyses of the WT mice revealed that more susceptible mice (i.e., those with lower $\mathrm{SI}$ ratios) exhibited higher methylation levels in Drd2 and $\mathrm{Nr} 3 \mathrm{c} 1$ in the PFC, and in Drd2 in the AMY, but lower methylation levels in Stmn 1 in the AMY. Taken together, these findings suggest that stress susceptibility was associated with higher methylation levels in Drd2 and/or Nr3c1, and lower methylation levels in Stmn1. In other words, the high levels of methylation in Drd2 and/or Nr3c1, and low levels of methylation in Stmn1, could be useful epigenetic markers of stress susceptibility, which partially accords with the results of the present study about the methylation levels in the three groups of WT and Stmn1 KO mice. In contrast, stress susceptibility in Stmn1 KO mice was associated with higher methylation levels in Drd2 and Stmn1 in the HIP. Although this finding regarding Drd2 is consistent with the result in WT mice, the finding regarding Stmn1 is opposite to the result in WT mice; moreover, this result is consistent with those described in the methylation levels in the three groups of WT and Stmn $1 \mathrm{KO}$ mice section. Although the reason why the Stmn $1 \mathrm{KO}$ mice exhibited an opposite pattern of methylation in Stmn1 remains unclear, it is possible that the methylation of Stmn1 in the Stmn1 KO SUS mice differed from that in the WT SUS mice because this process represents a compensatory mechanism against a pathophysiology that has already occurred in Stmn1 KO SUS mice [24], as described above.

The present study had several limitations that should be considered when interpreting the data. First, mRNA expression levels were not measured, so the implications of the methylation profiles can only be inferred indirectly. However, the primary aim of the present study was to explore the general nature of the methylation process for three key genes following social defeat stress. Second, only the results of the combined CpGs were presented. It has been suggested that a single, or only a few, critical CpG sites highly regulate gene expression $[25,26]$. However, because there were many $\mathrm{CpG}$ sites for each gene in the 
present study, and because opposite methylation responses were observed between different CpG sites, especially in Stmn1 (see Supplementary Tables 14-16, 20$22)$, it was difficult to present and appropriately interpret all of the data. Finally, the methylation data from Drd2 and Stmn 1 covering the regions upstream and downstream from the TSS were combined. There is controversy regarding the role that DNA methylation plays at promoters versus the gene body $[27,28]$, such that separate analyses might produce different results. However, the methylation patterns of the regions covering the upstream and downstream areas of the TSS were similar. The strength of the present study was that the overall methylation patterns of three key genes, in three critical brain regions, were explored in both WT and Stmn1 KO mice. Moreover, this study is the first to investigate DNA methylation using a social defeat stress paradigm.

In summary, social defeat stress in WT mice did not result in any changes in Drd2 methylation whereas, compared to the control group, significant hypermethylation was observed in $\mathrm{Nr} 3 \mathrm{c} 1$ in the HIP of the SUS group, and in Stmn1 in the AMY of the UNS group. Additionally, the methylation responses in Stmn1 KO mice differed from those in WT mice, such that there was evident hypermethylation in all three genes in both the SUS and UNS groups compared to the control group. Furthermore, comparison of the Stmn1 KO and WT mice in each group revealed identical patterns of hypermethylation in all three genes in the KO mice. Taken together, the present findings indicate that social defeat stress induced different epigenetic modifications in three genes among three groups of WT and Stmn1 KO mice. In particular, hypermethylation in $\mathrm{Nr} 3 \mathrm{C} 1$ in the HIP of the SUS group and in Stmn1 in the AMY of the UNS group, could serve as epigenetic biomarkers of stress susceptibility and stress resilience, respectively. These epigenetic changes may provide novel insights into the pathophysiology underlying defeat stress-related mental illnesses.

\section{- Funding}

This work was supported by the National Research Foundation of Korea (NRF) grant funded by the Korea government (MSIT) (No. 2020R1A2C2009413) and Fund of Biomedical Research Institute, Jeonbuk National University Hospital.

\section{Conflicts of Interest}

No potential conflict of interest relevant to this article was reported.

Author Contributions

Conceptualization: Young-Chul Chung. Data acquisition: Young-Eun Oh. Formal analysis: Young-Eun Oh, Thong Ba Nguyenm, Fatima Zahra Rami, Maryam Karamikheirabad. Funding: Young-Chul Chung. Supervision: Young-Chul Chung. Writing - original draft: Young-Eun Oh. Writingreview \& editing: Young-Chul Chung.

\section{ORCID}

Young-Eun Oh https://orcid.org/0000-0002-1365-5108

Thong Ba Nguyen https://orcid.org/0000-0001-9647-534X

Fatima Zahra Rami https://orcid.org/0000-0003-1706-6113

Maryam Karamikheirabad

https://orcid.org/0000-0002-6819-7511

Young-Chul Chung https://orcid.org/0000-0001-9491-1822

\section{SUPPLEMENTARY MATERIALS}

Supplementary data are available online.

\section{REFERENCES}

1. Roth TL, Lubin FD, Funk AJ, Sweatt JD. Lasting epigenetic influence of early-life adversity on the BDNF gene. Biol Psychiatry 2009;65:760-769.

2. Suderman M, McGowan PO, Sasaki A, Huang TC, Hallett MT, Meaney MJ, et al. Conserved epigenetic sensitivity to early life experience in the rat and human hippocampus. Proc Natl Acad Sci U S A 2012;109(Suppl 2):17266-17272.

3. Murgatroyd C, Patchev AV, Wu Y, Micale V, Bockmühl Y, Fischer $\mathrm{D}$, et al. Dynamic DNA methylation programs persistent adverse effects of early-life stress. Nat Neurosci 2009;12: 1559-1566.

4. Elliott E, Ezra-Nevo G, Regev L, Neufeld-Cohen A, Chen A. Resilience to social stress coincides with functional DNA methylation of the Crf gene in adult mice. Nat Neurosci 2010;13:1351-1353.

5. Missale C, Nash SR, Robinson SW, Jaber M, Caron MG. Dopamine receptors: from structure to function. Physiol Rev 1998;78:189-225.

6. Pani L, Porcella A, Gessa GL. The role of stress in the pathophysiology of the dopaminergic system. Mol Psychiatry 2000; 5:14-21.

7. Zhang TY, Labonté B, Wen XL, Turecki G, Meaney MJ. Epigenetic mechanisms for the early environmental regulation of hippocampal glucocorticoid receptor gene ex- 
pression in rodents and humans. Neuropsychopharmacology 2013;38:111-123.

8. Shumyatsky GP, Malleret G, Shin RM, Takizawa S, Tully K, Tsvetkov E, et al. Stathmin, a gene enriched in the amygdala, controls both learned and innate fear. Cell 2005;123:697709.

9. Brocke B, Lesch KP, Armbruster D, Moser DA, Müller A, Strobel A, et al. Stathmin, a gene regulating neural plasticity, affects fear and anxiety processing in humans. Am J Med Genet 2010;153B:243-251.

10. Nguyen TB, Prabhu VV, Piao YH, Oh YE, Zahra RF, Chung YC. Effects of stathmin 1 gene knockout on behaviors and dopaminergic markers in mice exposed to social defeat stress. Brain Sci 2019;9:215.

11. Liedtke W, Leman EE, Fyffe RE, Raine CS, Schubart UK. Stathmin-deficient mice develop an age-dependent axonopathy of the central and peripheral nervous systems. Am J Pathol 2002;160:469-480.

12. Krishnan V, Han MH, Graham DL, Berton O, Renthal W Russo SJ, et al. Molecular adaptations underlying susceptibility and resistance to social defeat in brain reward regions. Cell 2007;131:391-404.

13. Jin HM, Shrestha Muna S, Bagalkot TR, Cui Y, Yadav BK, Chung YC. The effects of social defeat on behavior and dopaminergic markers in mice. Neuroscience 2015;288:167-177.

14. Kember RL, Dempster EL, Lee TH, Schalkwyk LC, Mill J, Fernandes C. Maternal separation is associated with strainspecific responses to stress and epigenetic alterations to $\mathrm{Nr} 3 \mathrm{Cl}$, Avp, and Nr4a1 in mouse. Brain Behav 2012;2:455-467.

15. England R, Pettersson M. Pyro Q-Cp $C^{T M}$ : quantitative analysis of methylation in multiple CpG sites by Pyrosequencing ${ }^{\mathbb{R}}$. Nat Methods 2005;2:i-ii.

16. Brenet $\mathrm{F}$, Moh M, Funk P, Feierstein E, Viale AJ, Socci ND, et al. DNA methylation of the first exon is tightly linked to transcriptional silencing. PLoS One 2011;6:e14524.

17. Jones PA. Functions of DNA methylation: islands, start sites, gene bodies and beyond. Nat Rev Genet 2012;13:484-492.

18. Weaver IC, Cervoni N, Champagne FA, D'Alessio AC, Sharma
$\mathrm{S}$, Seckl JR, et al. Epigenetic programming by maternal behavior. Nat Neurosci 2004;7:847-854.

19. Bockmühl Y, Patchev AV, Madejska A, Hoffmann A, Sousa JC, Sousa $\mathrm{N}$, et al. Methylation at the CpG island shore region upregulates $\mathrm{Nr} 3 \mathrm{C} 1$ promoter activity after early-life stress. Epigenetics 2015;10:247-257.

20. Dent EW. Of microtubules and memory: implications for microtubule dynamics in dendrites and spines. Mol Biol Cell 2017:28:1-8.

21. Meaney MJ, Szyf M, Seckl JR. Epigenetic mechanisms of perinatal programming of hypothalamic-pituitary-adrenal function and health. Trends Mol Med 2007;13:269-277.

22. Mueller BR, Bale TL. Sex-specific programming of offspring emotionality after stress early in pregnancy. J Neurosci 2008; 28:9055-9065.

23. Martel G, Hevi C, Wong A, Zushida K, Uchida S, Shumyatsky GP. Murine GRPR and stathmin control in opposite directions both cued fear extinction and neural activities of the amygdala and prefrontal cortex. PLoS One 2012;7:e30942.

24. Han F, Jiang J, Ding J, Liu H, Xiao B, Shi Y. Change of Rin1 and stathmin in the animal model of traumatic stresses. Front Behav Neurosci 2017;11:62.

25. Zou B, Chim CS, Zeng H, Leung SY, Yang Y, Tu SP, et al. Correlation between the sing/e-site CpG methylation and expression silencing of the XAF1 gene in human gastric and $\mathrm{CO}$ Ion cancers. Gastroenterology 2006;131:1835-1843.

26. Cuomo M, Keller S, Punzo D, Nuzzo T, Affinito O, Coretti L, et al. Selective demethylation of two CpG sites causes postnatal activation of the Dao gene and consequent removal of D-serine within the mouse cerebellum. Clin Epigenetics 2019;11:149.

27. Yang X, Han H, De Carvalho DD, Lay FD, Jones PA, Liang G. Gene body methylation can alter gene expression and is a therapeutic target in cancer. Cancer Cell 2014;26:577-590.

28. Anastasiadi D, Esteve-Codina A, Piferrer F. Consistent inverse correlation between DNA methylation of the first intron and gene expression across tissues and species. Epigenetics Chromatin 2018:11:37. 